\title{
General discussion summary
}

This last general discussion session was chaired by P. L. Dutton (Department of Biochemistry/Biophysics, University of Pennsylvania, PA, USA: a visitor at Inorganic Chemistry Laboratory, University of Oxford, UK) and was initiated by S. Styring (Department of Biochemistry, Lund University, Lund, Sweden) addressing a question originally raised by D. Britt (Department of Chemistry, University of California, Davis, CA, USA) regarding the oxidation states of $\mathrm{Mn}$ in the Ruthenium- $\left(\mathrm{Mn}^{2+}\right)_{2}$ complex described in L. Hammarström's presentation. He could now confirm that a $\mathrm{Mn}^{3+}$ state was intermediate in the oxidation of the $\left(\mathrm{Mn}^{2+}\right)$ moiety when the buffer was changed from $\mathrm{Mes}$ to water.

P. L. Dutton asked T. A. Moore (Department of Chemistry and Biochemistry and Center for the Study of Early Events in Photosynthesis, Arizona State University, Tempe, AZ, USA) about the mechanism by which his system reached a ceiling of $4.4 \mathrm{kcal}(\mathrm{ca} .200 \mathrm{mV})$. T. A. Moore assumed that at a $\Delta \psi \cong 200 \mathrm{mV}$ or $\Delta \mathrm{pH}=3.1$ there was a breakdown in the barrier, although below this threshold the leak was minimal. The molecular mechanism for the transition was unknown and they plan to do more systematic experiments using different lipids and changing conditions to generate larger potential gradients.

The question of distance and the effect of high redox potentials on electron transport rates was raised by C. Zhang (Department of Biochemistry, Lund University, Lund, Sweden). Regarding distance measurements, P. L. Dutton explained that their approach was empirical; they simply matched measured and calculated rates. When the electron transfer was between metals such as in the $\mathrm{Ru}-\mathrm{Cu}$ complexes of $\mathrm{H}$. Gray, metal-to-metal distances worked well. However, for heams and chlorins, edge-to-edge distances were better than metal-metal. If a measured rate did not match the calculated rate then it may indicate that the distance used was incorrect. A classical case of this was the cyt $b c$ complex, where the theory predicted that the Fe-S centre of the Rieski subunit needed to be physically moved to allow rapid electron transfer to its acceptors.

P. Fromme (Max-Volmer-Laboratorium für Biophysikalische Chemie und Biochemie, Technische Universität Berlin, Berlin, Germany) asked T. A. Moore whether it was possible to generate ATP with his reconstituted ATPsynthase system using either a pure $\Delta \mathrm{pH}$ or pure $\Delta \psi$. T. A. Moore was not sure, but had found that some $\Delta \psi$ seemed to be advantageous. Again more experiments are needed, he said.

Finally C. Dismukes (Department of Chemistry, Princeton University, Princeton, NF, USA) raised the issue that the structure of PSII reported by P. Fromme may be distorted by the effect of X-ray damage. P. Fromme thought this unlikely, explaining that they frequently moved the crystal in the X-ray beam so as to collect the data from fresh regions. Of course, as she explained, after intense X-ray radiation there will be damage, but under these conditions no diffraction data can be collected.

\section{GLOSSARY}

ATP: adenosine triphosphate

PSII: photosystem II 